\title{
The simulation of bat and dolphin signals and the estimate of their vocal tract shapes
}

\author{
Eiichi Kashiwagi and Yoichi Okamoto \\ Department of Applied Physics, National Defense Academy, \\ Yokosuka, 239, Japan
}

(Received 28 April 1987)

\begin{abstract}
The signals maximizing the wide-band ambiguity function, in which the effects of both phase-fluctuation and Doppler shift are taken into account, are derived by using techniques of variational calculus. These signals are found to be very similar to some of the waveforms used by dolphins and bats for echolocation. It is indicated that these signals can be also produced by changing only the coefficients of the derived differential equation. Their vocal tract shapes are estimated from these theoretical signals by applying linear prediction techniques and found to resemble the configuration of human whistling.
\end{abstract}

PACS number: 43. 30. Bp, 43. 60. $\mathrm{Cg}$, 43. 80. Lb

\section{INTRODUCTION}

Echo signals are inevitably distorted by the effects of phase-fluctuation and Doppler shift, suffered during the propagation and further disturbed by noise. It has been demonstrated that a correlation process is a sufficient statistic for the detection of a signal in white Gaussian noise.

This paper attempts to find the optimal waveforms for phase-fluctuation tolerance and Doppler tolerance, i.e., signals such that magnitude of correlator output is relatively unaffected by the effects of phasefluctuation and Doppler shift. Theoretical consideration is given to derive the signal maximizing the wideband ambiguity function in which the effects of both phase-fluctuation and Doppler shift are taken into account. Wide-band ambiguity function analysis is combined with techniques of variational calculus. With appropriate constraints for making physically realizable waveforms, the derived signals are found to be very similar to waveforms used by dolphins and bats for echolocation. It is indicated that these signals can be produced by changing only the coefficients of the differential equation, while dolphins and bats perform their echolocation under different environments, i.e., the former in underwater and the latter in the air. This leads us to expect the similarity of their signal-producing mechanism and signalprocessing system.

By the way, the simulated bat and dolphin signals have been indicated before. ${ }^{1,2)}$ But these signals were theoretically derived by maximizing the ambiguity function in which only Doppler shift effect was taken into account. Then the simulated bat signals were obtained by passing the derived signals through a highpass filter of an exponential type ${ }^{1)}$ and so not directly derived from the solved equation. One of the reasons can be presumed to be that the phase-fluctuation effect considered in this paper is not included in the ambiguity function.

Finally, using the derived theoretical signals, the vocal tract shapes of dolphins and bats are estimated by applying linear prediction techniques discussed in many publications on speech analysis.

\section{PHASE-FLUCTUATION AND DOPPLER TOLERANT SIGNALS ${ }^{3,4)}$}

A signals $u(t)$ with spectrum $U(\omega)$ is described by using Analytic Signal representation, which defines $U(\omega)=0$ for $\omega<0$. Then, the time signal $u(t)$ is the inverse Fourier transform of the resulting one-sided spectrum and the transmitted signal $u_{\mathrm{R}}(t)$ can be 
written as the real part of $u(t)$ by defining $U(-\omega)=$ $U^{*}(\omega)$, where the asterisk indicates a complex conjugation.

$$
\begin{aligned}
u(t) & =\frac{1}{2 \pi} \int_{0}^{\infty} U(\omega) e^{j \omega t} d \omega \\
& =a(t) e^{j \theta(t) \quad(\dot{\theta}(t)>0)} \\
u_{\mathrm{R}}(t) & =a(t) \cos \theta(t) .
\end{aligned}
$$

Here, it can be provided that much more detection degradation due to the fluctuation suffered during the propagation results from the phase-fluctuation effect than from the signal-envelope distortion effect, because the fluctuation of the envelope $a(t)$ is sufficiently nonoscillatory. So, only the phase term is taken into account for fluctuation effects in analyzing.

When the deviation $\varepsilon(t)$ from mean propagation time at time $t$ results from the fluctuation effect, the phase lag is described as $\exp [-j \omega \varepsilon(t)]$. Then the echo signal $v(t)$ distorted by the effects of both phasefluctuation and Doppler shift can be represented as

$$
v(t)=\frac{1}{2 \pi \sqrt{s}} \int_{0}^{\infty} U\left(\frac{\omega}{s}\right) e^{j \omega(t-\varepsilon(t))} d \omega
$$

where $s$ is the Doppler factor.

If we assume that $\varepsilon(t)$ obeys a Gaussian random process with zero mean and variance $\sigma^{2}$, the ensemble average for Eq. (3) can be obtained as

$$
E[v(t)]=\frac{1}{2 \pi \sqrt{s}} \int_{0}^{\infty} U\left(\frac{\omega}{s}\right) e^{-\sigma^{2} \omega^{2} / 2} e^{j \omega t} d \omega
$$

through the result that the characteristic function becomes

$$
E\left[e^{-j \omega \varepsilon(t)}\right]=e^{-\sigma^{2} \omega^{2} / 2} .
$$

Accordingly, the cross-correlation function $A_{\mathrm{u}}(\tau, s$, $\sigma$ ) between the transmitted signal and the echo signal can be written as

$$
\begin{aligned}
A_{\mathrm{u}}(\tau, s, \sigma)= & \frac{1}{2 \pi \sqrt{s}} \int_{0}^{\infty} U\left(\frac{\omega}{s}\right) U^{*}(\omega) \\
& \times e^{-\sigma^{2} \omega^{2} / 2} e^{j \omega \tau} d \omega .
\end{aligned}
$$

The ambiguity function $\left|A_{\mathrm{u}}\right|^{2}$ is investigated by reason of the fact that the envelope of the matched filter response is used for the detection. It has been known that the $\left|A_{\mathrm{u}}(\tau, s, 0)\right|^{2}$ function has a maximum at $(\tau$, $s)=(0,1)$ and that the constant amplitude contours very near $(0,1)$ are elliptical for all finite energy signals. A three-dimensional Taylor series describes the behavior of $A_{\mathrm{u}}$ near $(\tau, s, \sigma)=(0,1,0)$. So we take a second-order approximate expression under as- sumption $|\tau| \ll 1,|s-1| \ll 1$ and $\sigma \omega<1$. Then, the derived expression takes the maximum value $\left|A_{\mathrm{u}}\left(\tau_{\mathrm{m}}, s, \sigma\right)\right|^{2}$ in $\tau=\tau_{\mathrm{m}}$ giving $\partial\left|A_{\mathrm{u}}\right|^{2} / \partial \tau=0$ for given $s$ and $\sigma$.

As the result, the derivation of $U(\omega)$ maximizing the $\left|A_{\mathrm{u}}\left(\tau_{\mathrm{m}}, s, \sigma\right)\right|^{2}$ function gets equivalent to finding $U(\omega)$ minimizing the following Eq. (7).

$$
\begin{aligned}
I= & \sigma^{2} \cdot \frac{1}{2 \pi} \int_{0}^{\infty} \omega^{2}|U(\omega)|^{2} d \omega \\
& +(s-1)^{2}\left(\frac{1}{2 \pi} \int_{0}^{\infty} \omega^{2}|\dot{U}(\omega)|^{2} d \omega\right. \\
& \left.-\left|\frac{1}{2 \pi} \int_{0}^{\infty} \omega \dot{U}(\omega) U^{*}(\omega) d \omega\right|^{2}\right)
\end{aligned}
$$

where $U(\omega)$ is normarized to unit energy and $\dot{U}(\omega)$ denotes the differentiation with respect to $\omega$.

In Eq. (7), the first term means the detection degradation resulting from the phase-fluctuation effect and the second term means the degradation from the Doppler shift effect discussed in Ref. 2). Accordingly, the total detection degradation can be described as the addition of respective detection degradations. Here, in order to produce physically realizable signals, the following constraints, i.e., energy, center frequency and mean-square time duration, will be required:

$$
\begin{aligned}
& \frac{1}{2 \pi} \int_{0}^{\infty}|U(\omega)|^{2} d \omega=\int_{-\infty}^{\infty} a^{2} d t=1 \\
& \frac{1}{2 \pi} \int_{0}^{\infty} \omega|U(\omega)|^{2} d \omega=\omega_{\mathrm{c}} \\
& \int_{-\infty}^{\infty} t^{2}|u(t)|^{2} d t=\int_{-\infty}^{\infty} t^{2} a^{2} d t=D_{t}{ }^{2} .
\end{aligned}
$$

The calculus of variation is applied to find the optimal waveforms for phase-fluctuation tolerance and Doppler tolerance, using the three constraints shown above.

We use Lagrange multipliers $\lambda_{\mathrm{E}}$ for the energy constraint, $\lambda_{c}$ for the center frequency one and $\lambda_{t}$ for the duration one. Then, the following functional, in which the representation is transformed in time region, is obtained.

$$
\begin{aligned}
J(\dot{a}, \dot{\theta}, a)= & \int_{-\infty}^{\infty}\left[\left(\left(\lambda_{\mathrm{E}}-\frac{1}{4}\right)+\lambda_{\mathrm{c}} \dot{\theta}+\left(t^{2}+R^{2}\right) \dot{\theta}^{2}\right.\right. \\
& \left.\left.+\lambda_{\mathrm{t}} t^{2}\right) a^{2}+\left(t^{2}+R^{2}\right) \dot{a}^{2}\right] d t \\
& -\left(\int_{-\infty}^{\infty} t a^{2} \dot{\theta} d t\right)^{2}
\end{aligned}
$$




\section{E. KASHIWAGI and Y. OKAMOTO: THE SIMULATION OF BAT AND DOLPHIN SIGNALS}

where

$$
R=\frac{\sigma}{|s-1|} .
$$

Because the Euler-Lagrange equation cannot be used for Eq. (11), techniques of variational calculus are directly applied. As the result, the following differential equations are provided for $a(t)$ and $\theta(t)$.

$$
\begin{aligned}
\ddot{a}= & -\frac{2 t}{t^{2}+R^{2}} \dot{a}+\left(\alpha+\frac{\beta}{t^{2}+R^{2}}\right. \\
& \left.-\frac{2 \gamma k t}{\left(t^{2}+R^{2}\right)^{2}}+\frac{k^{2} R^{2}-\gamma^{2}}{\left(t^{2}+R^{2}\right)^{2}}\right) a \\
\dot{\theta}= & \frac{k t+\gamma}{t^{2}+R^{2}}
\end{aligned}
$$

where

$$
\begin{aligned}
& k=\int_{-\infty}^{\infty} t a^{2} \dot{\theta} d t \\
& \alpha=\lambda_{\mathrm{t}} \\
& \beta=\lambda_{\mathrm{E}}-\frac{1}{4}-\lambda_{\mathrm{t}} R^{2}-k^{2} \\
& \gamma=-\frac{\lambda_{\mathrm{c}}}{2} .
\end{aligned}
$$

From Eq. (14), $\theta(t)$ becomes

$$
\theta(t)=\frac{k}{2} \cdot \log \left(t^{2}+R^{2}\right)+\frac{\gamma}{R} \tan ^{-1} \frac{t}{R}+\theta_{0}
$$

where $\theta_{0}$ denotes a constant.

For $t \gg R$ and $t \gg \gamma / k, \dot{\theta}(t)$ can be approximated as

$$
\dot{\theta}(t) \fallingdotseq \frac{k}{t} .
$$

This is called a linear period modulation signal and it has been known that some kinds of bats and dolphins use this type of signal in their "sonar."

\section{SIMULATED BAT AND DOLPHIN SIGNALS}

Some of the sonar signals that are used by dolphins and bats can be modelled with Eqs. (13) and (19). The waveforms similar to sonar pulses used by the dolphin (Tursiops) and the bat (Myotis lucifugus) ${ }^{1)}$ are shown in Figs. 1 and 2, respectively, for parameters $k, R, \alpha, \beta, \gamma$, and $\theta_{0}$ given in the figure captions. $\theta_{0}$ corresponds to the initial phase value.

Of these parameters, $k$ can be estimated from Eq. (20) if the frequency range of the signal and its pulse length are known. We also notice from Eq. (12) that
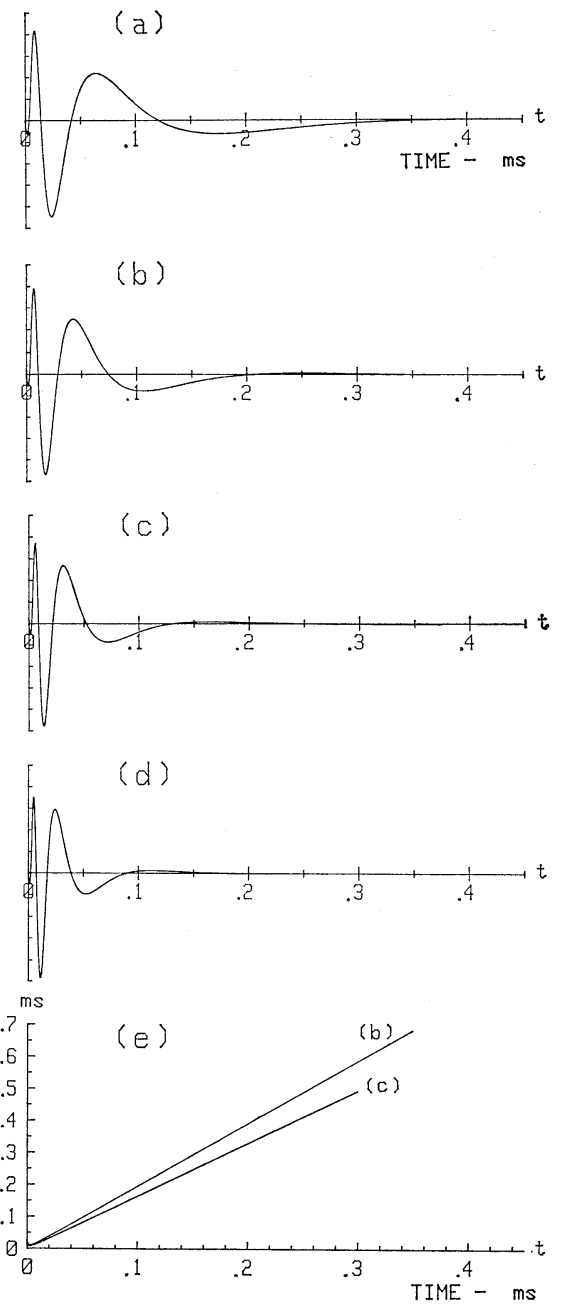

Fig. 1 Time signals $u_{\mathrm{R}}(t)$ derived from Eqs. (13) and (19), which are similar to those used by the dolphin (Tursiops). Parameters $\left(k, R, \alpha, \beta, \gamma, \theta_{0}\right)$ are taken as (a) (2.9, $0.048,0.1,-0.28,0.041,-0.1 \pi)$, (b) (3.2, $0.042,0.45,-0.22,0.041,-1.68 \pi)$, (c) $(3.5,0.038,1.52,-0.21,0.0407,-1.3 \pi)$ and (d) $(3.8,0.035,3.58,-0.21,0.0405$, $-0.92 \pi)$, where (e) represents the instantaneous period in signals (b) and (c).

whether $R$ is taken as large or small depends on whether bats and dolphins make much effort to suppress the phase-fluctuation effect or the Doppler shift effect, in their signal production. Figures 1 and 2 show that the value of $R$ in the simulated waveforms for the bat is larger than $R$ for the dolphin. Namely, bats can be supposed to produce signals 

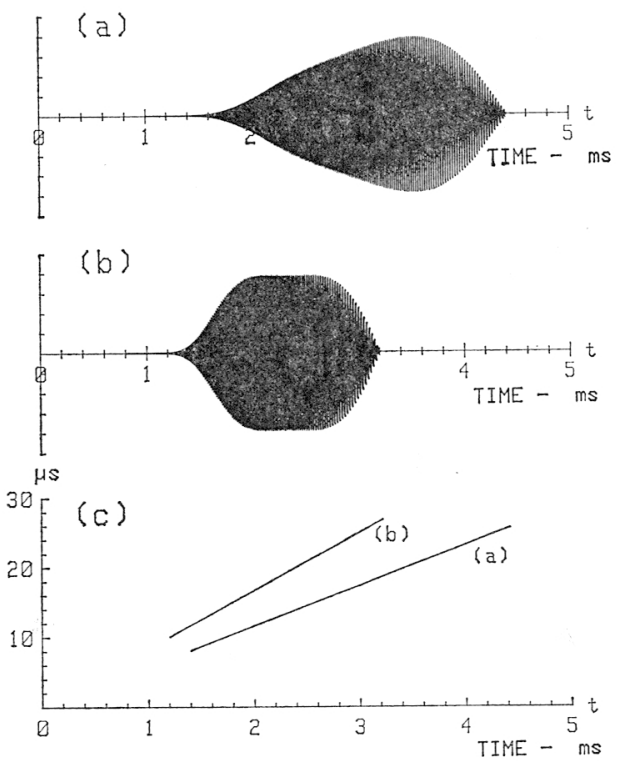

Fig. 2 Time signals $u_{\mathrm{R}}(t)$, which are similar to (a) cruising pulse and (b) pursuit pulse of the bat (Myotis lucifugus). Parameters $(k, R, \alpha, \beta, \gamma)$ are taken as (a) $(1,080,0.08$, $-50,2,142,3.5)$ and (b) (750, 0.15, $-219.2,5,928,10.965)$, where (c) represents the instantaneous period in signals (a) and (b).

paying much more attention to the suppression of the phase-fluctuation effect than dolphins.

Figures 1 (e) and 2 (c) show the instantaneous period of the waveforms. In these figures the simulated signals are almost perfectly linear period modulated because of the very small value of $R$ and $\gamma / k$, i.e., $\theta(t) \fallingdotseq t / k$.

Figures 3 (a) and (b) show the power spectra of the signals in Figs. 1 (b) and (c), respectively. Figures 4 (a) and (b) also show those of signals in Figs. 2 (a) and (b), respectively. They show that the spectrum of dolphin signals ranges to at least three octaves and that of bat signals to one octave. These spectra contain no harmonics but only the fundamental, in agreement with the result analyzed from the recorded data. ${ }^{5)}$ On the other hand, the human voice in vowels has harmonics covering a wide band of frequencies.

It is also noted that dolphins and bats generate sound signals having much larger time-band width products than the man-made radar or sonar.
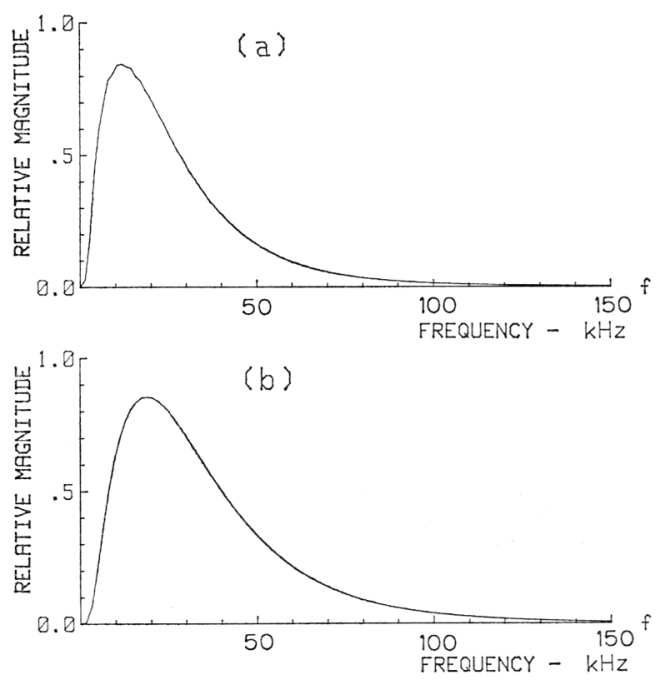

Fig. 3 The power spectra of the dolphin signals, which range to at least three octaves. (a) and (b) correspond to Figs. 1 (b) and (c), respectively.
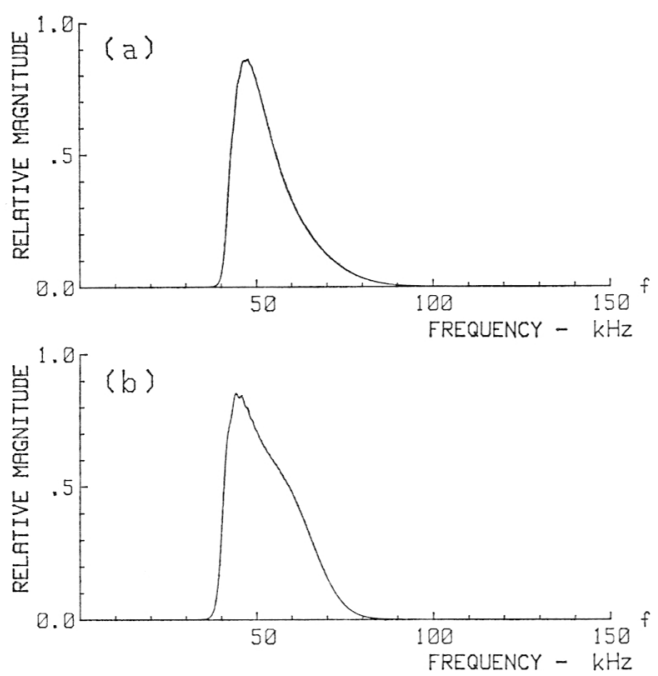

Fig. 4 The power spectra of the bat signals, which range to one octave. (a) and (b) correspond to Figs. 2 (a) and (b), respectively.

\section{VOCAL TRACT SHAPES OF BAT AND DOLPHIN}

It has been known that the human vocal tract shapes can be estimated from the speech wave by 


\section{E. KASHIWAGI and Y. OKAMOTO: THE SIMULATION OF BAT AND DOLPHIN SIGNALS}

applying linear prediction techniques, ${ }^{6)}$ namely, that the ratio of cross section area in the vocal tract can be estimated from the partial autocorrelation coefficients as follows:

$$
\frac{A_{i}}{A_{i+1}}=\frac{1+\kappa_{i}}{1-\kappa_{i}}
$$

where $A_{i}$ is the $i$ th cross section area and $\kappa_{i}$ the $i$ th coefficient of the partial autocorrelation.

Application of this techniques enables us to estimate the vocal tract shapes of dolphins and bats with their simulated signals. The order (step numbers in the area function) in linear prediction model is selected such as the model spectrum fits optimally the original one (Figs. 3 and 4).

Figures 5 and 6 show examples for the dolphin and the bat, respectively. Figures 5 (a) and (b) show the vocal tract shapes for the generation of the waveforms in Figs. 1 (b) and (c), respectively, and Figs. 6 (a) and (b) for the generation of the waveforms in Figs. 2 (a) and (b), respectively.

Here, the estimation of the vocal tract shapes is performed under the assumption that for the bat signals the transmission path of sound is a series of air-filled resonators just as for human speech, and that for the dolphin signals it is coupled by the matched acoustic impedance because of the quite

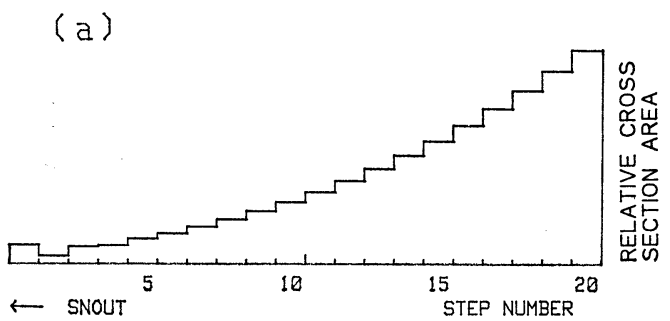

(b)

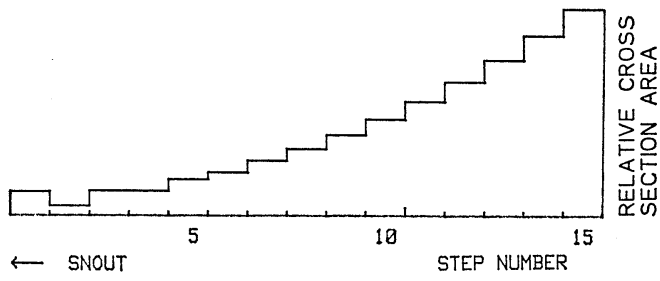

Fig. 5 The estimated vocal tract shapes of the dolphin, which lead us to guess that of a human being when he whistles. (a) and (b) are those for the signals in Figs. 1 (b) and (c), respectively.
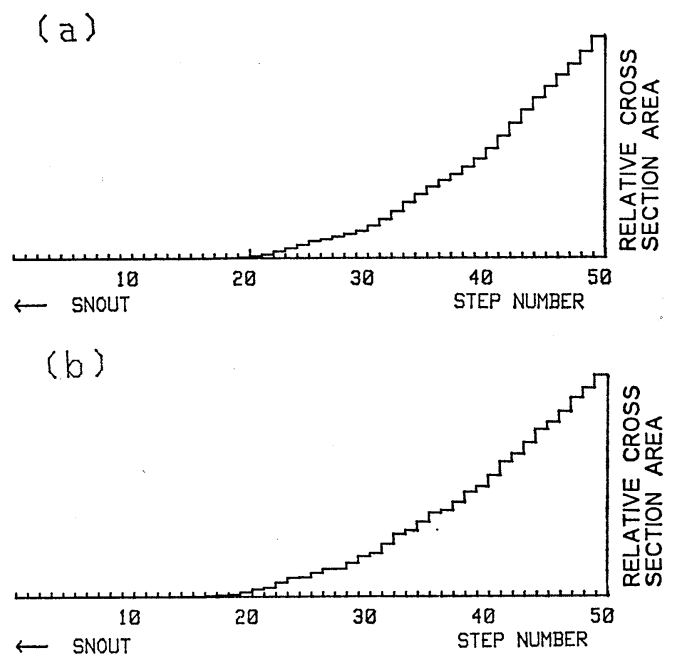

Fig. 6 The estimated vocal tract shapes of the bat. (a) and (b) are those for the signals in Figs. 2 (a) and (b), respectively.

similar acoustic impedance of muscle and water.

Both Figs. 5 and 6 give similar vocal tract shapes and lead us to guess a configuration corresponding to that of a human being when he whistles. These configurations also closely resemble the human vocal tract shape for a voiceless fricative consonant $\left[\int\right] .^{7)}$

\section{CONCLUSION}

Signals minimizing the detection degradation resulting from the effects of phase-fluctuation and Doppler shift have been derived for a system using correlation detection. These signals are found to be very similar to waveforms used by bats and dolphins for echolocation. By using these theoretical signals, we have estimated the power spectra of their signals and their vocal tract shapes. It is interesting to note that both bat signals and dolphin signals can be produced by changing only the coefficients of the differential equation derived under some assumed conditions. This suggests to us the similarity of their echolocation systems.

So far, no postulated sound-producing mechanism for dolphins or bats has given the adequate description for the complexities observed on the anatomical and morphological aspects. So we study their signalproducing and signal-processing mechanism from an engineering standpoint. The use of the theoretical signals will encourage us to do further work for the full understanding of their echolocation systems, 
which is essential to the improvement in performance of man-made radar and sonar.

\section{REFERENCES}

1) R. A. Altes and W. D. Reese, "Doppler-tolerant classification of distributed targets-A bionic sonar," IEEE Trans. Aerosp. Electron. Syst. AES11, 708-723 (1975).

2) R. A. Altes and E. L. Titlebaum, "Bat signals as optimally Doppler-tolerant waveforms," J. Acoust. Soc. Am. 48, 1014-1020 (1970).

3) E. Kashiwagi, "Optimally phase-fluctuation and Doppler tolerant waveforms for a system using correlation detection-Dolphin and bat signals," J.
Acoust. Soc. Jpn. (J) 40, 460-467 (1984) (in Japanese).

4) E. Kashiwagi, "Optimally phase-fluctuation tolerant waveforms for a system using correlation detection," J. Acoust. Soc. Jpn. (J) 40, 327-331 (1984) (in Japanese).

5) W. E. Evans, "Echolocation by marine delphinids and one species of fresh-water dolphin," J. Acoust. Soc. Am. 54, 191-199 (1973).

6) S. Saito and K. Nakata, Fundamentals of Speech Signals Processing (Ōmusha, Tokyo, 1981), p. 152 (in Japanese).

7) S. Masaki and K. Shirai, "Estimation of the vocal tract shape producing fricative consonants," J. Acoust. Soc. Jpn. (E) 5, 51-58 (1984). 